\title{
MÉTODO DE INTERVENCIÓN Y PROCESO DE TRA- MITACIÓN DE LAS ACTUACIONES EN ELEMENTOS DEL PATRIMONIO HISTÓRICO DE ANDALUCÍA
}

\author{
Juan Antonio Fernández \\ Naranjo \\ Arquitecto Jefe del \\ Departamento de Conservación \\ del Patrimonio Histórico de la \\ Delegación de la Consejería \\ de Cultura
}

La dilatada polémica y el amplio debate sobre el método en la restauración de los Bienes Culturales continua sin solución, siendo posible que no la tenga, y es adecuado y positivo que se mantenga vivo dentro de la propia disciplina como motivo de confrontación y discusión fructífera, de forma de que cada vez más esta materia se enriquezca con opiniones, corrientes y posturas diversas y, por qué no, con modas.

Al igual que con el método, el de los criterios es otro debate que se mantiene abierto, afortunadamente. Ambos, método y criterios deben repercutir en la manera de intervenir en el Patrimonio Histórico(l) y es la Administración, la Consejería de Cultura en nuestro caso, la que debe propiciar, por un lado, el que el debate no sólo se produzca, sino que se mantenga abierto y vivo, y por otro, garantizar que sus avances y conclusiones sean puestas en práctica, y así, una vez aplicadas y experimentadas, volver a incidir con los resultados en el propio debate, cerrando así el círculo de un proceso sin solución de continuidad.

Por todos es reconocido el carácter global del concepto Bien Cultural sin que quepa hacer distinciones dentro de él y por lo tanto la base teórica de partida es la misma. Correspondiendo a esta generalidad la adscripción de su tutela e intervención no corresponde a una sola disciplina: es pues la pluridisciplinareidad la única forma adecuada de enfocar las soluciones a los problemas planteados por el Patrimonio Histórico.

La Consejería de Cultura es la encargada de velar por la conservación y protección del Patrimonio Histórico en el ámbito de la Comunidad Autónoma de Andalucía(2) así se ha dotado de un cuerpo legislativo que ordena y regula la intervención en los Bienes Culturales sujetos a su tutela, creando toda una organización administrativa que actúa en dicha intervención(3).

Es fundamental que esta estructura garantice la multidisciplinareidad y que dé respuestas, a la vez que participa, en los debates del método y de los criterios. Ello no es posible, a mi entender, si no se mejora y adecua a la idea anterior el proceso de tramitación de las intervenciones en el Patrimonio Histórico.
En la actualidad, la intervención en elementos de interés histórico se viene haciendo bajo el apremio del día a día, sin que se puedan conocer bien los elementos en los que se actúa y aceptando las propuestas que los profesionales encargados de resolver el problema presentan. A esta circunstancia hay que unir la precaria situación de personal y medios en la que se encuentran las Delegaciones Provinciales y la propia Dirección General de Bienes Culturales.

También, hay que añadir a la situación actual, el hecho del funcionamiento independiente que llevan a cabo los distintos servicios de la referida Dirección General. En cuanto a la relación entre el Servicio de Conservación y Obras con el de Protección del Patrimonio Histórico llega a rozar la irregularidad administrativa, según han puesto de manifiesto los recientes Debates Internos sobre las Comisiones Provinciales del Patrimonio Histórico(4), al producirse las actuaciones directas de la Dirección General sin el preceptivo informe de dichas Comisiones. La relación entre el Servicio de Conservación y Obras con el de Investigación y Difusión no es completa ya que no está incluido o enlazado con el proceso de intervención, sino que se produce ocasionalmente y de forma independiente la una de la otra.

La idea de crear unidades de intervención directa sobre elementos del Patrimonio Histórico en las Delegaciones Provinciales, aparte de resultar una situación al menos utópica, no creo que sea una solución operativa ya que dada la complejidad de dichas actuaciones y la tecnología ya incorporada al proceso de proyecto, exigiría de la Administración un enorme esfuerzo en personal y medios. Además, este planteamiento solo sería solución para elementos reducidos en sus dimensiones y sin muchos problemas(5). Sí podría ser una ventaja propiciar estas unidades en las Diputaciones Provinciales y controlar su funcionamiento e intervenciones mediante Convenios dentro del ámbito de las autorizaciones previstas en la legislación vigente sobre Patrimonio Histórico.

Tampoco se puede pensar en mantener el modo de actuación actual incorporando nuevos elementos de control o inspección, ya que los existentes no se han puesto en marcha y duplicarlos no soluciona los problemas de base, los cuales dominarán 
el proceso a la larga y harán imposible la tutela de los tuteladores.

Hay que pensar pues que la solución está en incorporar los razonamientos y el estado del debate método-criterios al proceso administrativo, el cual debe entender la actuación en el patrimonio histórico como un todo, desde los planteamientos iniciales que aconsejan su programación y marcan la estrategia o líneas de actuación (directa, convenio, subvención, orden de ejecución, etc.) (6), hasta su difusión y conocimiento público, no solo de los resultados, sino de todo el proceso, que debe ser escrupulosamente limpio administrativamente y debería cumplir con todas las condiciones y estipulaciones que las leyes y reglamentos que esta Consejería ha propiciado para regular la intervención en el Patrimonio Histórico.

Son las Delegaciones Provinciales las que mejor pueden garantizar la idoneidad del proceso anterior ya que en su ámbito es donde mejor se reúne el conocimiento directo e inmediato de los problemas con las condiciones administrativas que permiten, en igualdad, el cumplimiento de la Ley, en cuanto que en ellas están residenciadas las Comisiones Provinciales de Patrimonio Histórico(7) y a su vez se encargan del seguimiento de la ejecución material de las referidas actuaciones.

Dentro de las Delegaciones Provinciales es función de los Departamentos de Conservación del Patrimonio Histórico el llevar a cabo la mencionada gestión, y en este sentido creo que habría que tomar las siguientes medidas, que elevo en forma de propuesta, considerando, como ya se ha dicho, que la actuación sobre el Patrimonio Histórico empieza con los planteamientos previos y culmina con su difusión, entendiendo la restauración como la recuperación de unos reconocidos valores culturales y colectivos que se devuelven a su uso, disfrute y conocimiento a la sociedad que debe considerar, a partir de ese momento, prioritario su mantenimiento y conservación:

A. Una vez programada una intervención por la Dirección General a propuesta de la Delegación Provincial, el Departamento de Conservación planteará las líneas a seguir, siendo funda- mental una adecuada estrategia de estudios previos (ensayos sobre su patología, de sus materiales, planimetría, historiografía, arqueología, etc.). La coordinación de estos trabajos desde el mencionado Departamento garantizaría la unidad de acción de las diversas disciplinas que intervienen en el proceso.

B. El conjunto de los resultados de los estudios previos conforman un estado de conocimiento bastante amplio de la problemática de la intervención que ayuda a marcar prioridades, y a confirmar o corregir las líneas de actuación de partida.

C. Marcadas las prioridades, y teniendo en cuenta las líneas de actuación, se encarga a un profesional la solución concreta del problema mediante la redacción del correspondiente proyecto de restauración, redacción que será estrechamente seguida por el Departamento de Conservación.

D. Redactado el proyecto, y previo informe del referido Departamento, dicho Proyecto debe ser sometido a informe de la Comisión Provincial del Patrimonio Histórico, en cumplimiento del trámite de autorización de las actuaciones en Patrimonio Histórico recogida en la legislación vigente(8).

E. El Proyecto, acompañado de los informes favorables del Departamento de Conservación y de la Comisión Provincial de Patrimonio Histórico, se remitirá a la Dirección General para su supervisión técnica y posterior autorización ${ }^{(9)}$.

F. Puesta en marcha la obra, el Departamento de Conservación no se limitará al seguimiento administrativo de la misma, sino que debe entenderse que la Dirección Facultativa está bajo su coordinación ${ }^{(10)}$. Hay que tener en cuenta que la existencia de los trabajos previos reduce con mucho las posibilidades de desviaciones entre el Proyecto y las obras, al tener un amplio conocimiento del elemento en que se interviene y reduciendo bastante la aparición de Modificados.

\section{Por todos es reconocido el carácter global del concepto de Bien Cultural sin que quepa bacer distinciones dentro de él, por lo tanto la base teórica de partida es la misma.}

En correspondencia con ello la adscripción de su tutela e intervención no corresponde a una sola disciplina: es pues la pluridisciplinareidad la unica forma adecuada de enfocar las soluciones a los problemas planteados por el Patrimonio Histórico 


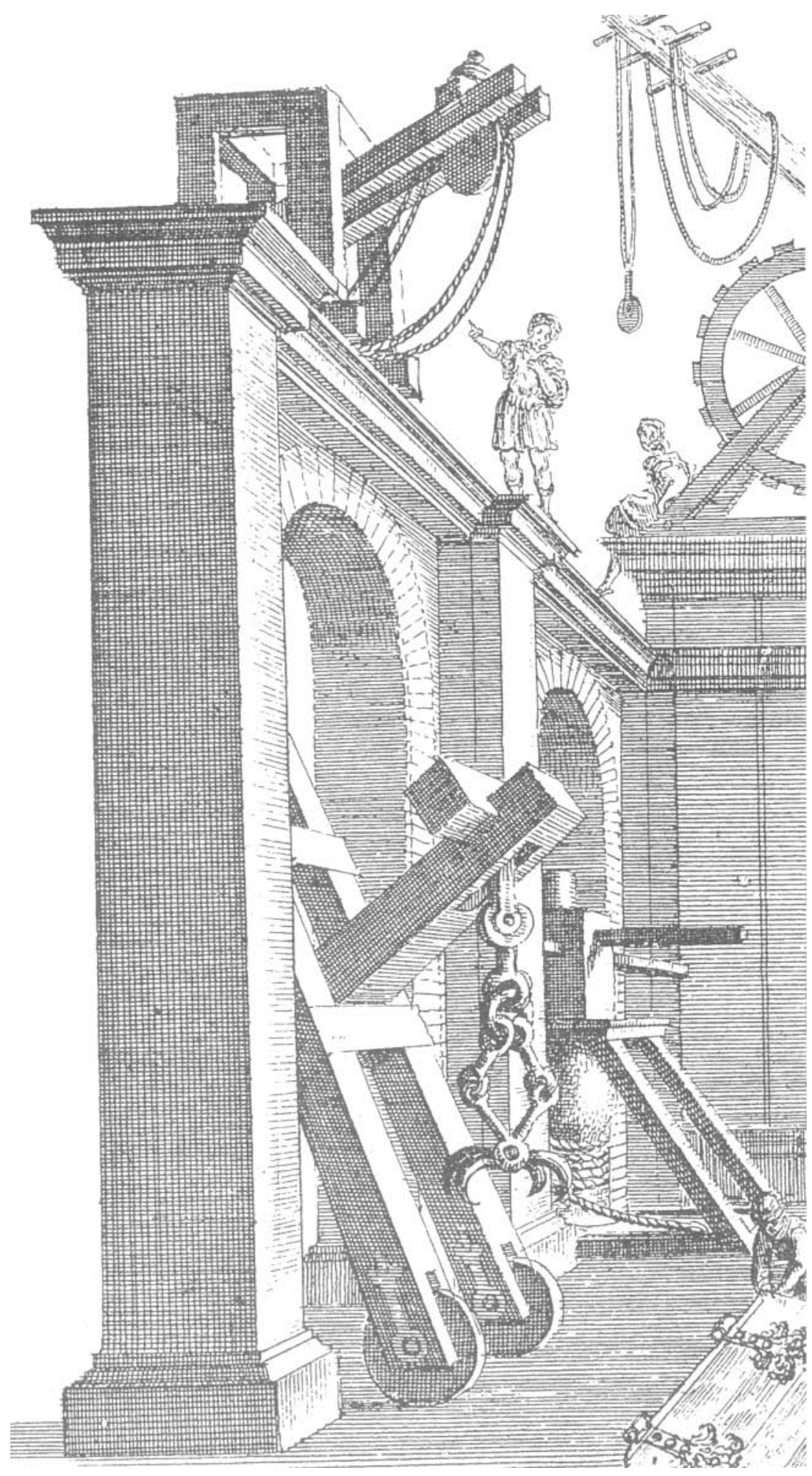

G. Finalizadas las obras se redactará una memoria final con amplia documentación gráfica, que pasará a formar parte y completará el nivel de conocimiento dado por los estudios

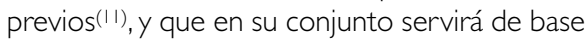
para la difusión de los resultados y divulgación del método.

H. Correspondería a la Dirección General de
Bienes Culturales, a partir- de aquí, la implantación y coordinación de todo el sistema en el territorio de la Comunidad Autónoma, la puesta al día e intercambio de experiencias de las distintas Delegaciones Provinciales, y su traslado al campo disciplinar de la Restauración Monumental mediante las consiguientes Jornadas, Simposium, etc., así como la participación como Administración en encuentros del mismo tipo organizados en otras Comunidades e incluso en el extranjero.

Considero, pues, que con este proceso cumplimos con los siguientes objetivos:

I. Garantizar a través de los estudios previos y de los propios Departamentos de Conservación la pluridisciplinareidad. Para ello hay que pensar en adecuar: no solo la estructura del mismo de forma que cuente, al menos, con las disciplinas básicas (arquitectura, arqueología, historia del arte y restauración mueble), sino los medios necesarios.

2. Garantizar el estricto cumplimiento de la legislación vigente en cuanto a autorizaciones de las intervenciones en el Patrimonio Histórico, en base a una estructura administrativa establecida que participa en el momento adecuado de la tramitación, con lo que se constituye en parte integrante del equipo que es capaz de ejercer una acción sobre el Patrimonio Histórico de su ámbito territorial.

3. Optimizar la gestión y los resultados: el amplio conocimiento que se obtendría del problema a través de los estudios previos, haría que la Administración se formase un criterio adecuado para la intervención, que acompañado de la correspondiente coordinación del proyecto, de la ejecución de las obras y del cuerpo de documentación final, lograrían que la Consejería de Cultura pudiera ser reconocida como autora de la actuación, ya que no sólo financiaría y gestionaría, sino que también aplicaría un método y fijaría criterios, incorporándose así al debate interdisciplinar con un cómputo importante de intervenciones no sólo por su número y presupuesto, sino por la calidad de los resultados y las aplicaciones científicas y metodológicas, así como por la recuperación e incorporación a la sociedad a la que pertenece, que es, a su vez, la que demanda los resultados satisfactorios.

Todo lo anterior debe entenderse como aportación a la racionalización del proceso de intervención en los elementos históricos, ámbito que, dentro de la actividad de la Dirección General, se ha quedado bloqueado y limitado al encargo, supervisión y ejecución de las obras del Proyecto. Sería oportuno que en base a la situación actual, y si pudiera ser al contenido de este escrito, se plantee un debate que llegue al procedimiento idóneo para conseguir que la conservación del Patrimonio Histórico de nuestra Comunidad, llegue a ser reconocida como una labo de la Administración, y como aportación que ésta debe hacer a la restauración de los Bienes Culturales. 


\section{NOTAS}

I. Hacer referencia aquí al completo y brillante análisis que Antón Capitel hace en su libro "Metamorfosis de los monumentos y teorías de la restauración". Alianza Editorial, S. A. Madrid 1988.

2. Un dudoso entendimiento del concepto de Bien Cultural y de la idea de Patrimonio Histórico ha llevado a permitir que la Consejería de Obras Públicas, a través de determinados programas, esté interviniendo en elementos del Patrimonio Histórico, sin método y con un criterio muy distinto al que pudiera aplicarse en la de Cultura. Véase el catálogo de la exposición "Arquitectura Pública en Andalucía" editado recientemente.

3. Ley |//99|, de 3 de junio, del Patrimonio Histórico de Andalucía.

Decreto 4/1993, de 26 de enero, Reglamento de Organización Administrativa del Patrimonio Histórico.

Decreto 32/1993, de 16 de marzo, Reglamento de Actividades Arqueológicas.

Decreto 19/1995, de 7 de febrero, Reglamento de Protección y Fomento del Patrimonio Histórico.

4. Estos Debates Internos han sido promovidos por la Dirección General de Bienes Culturales a través del Instituto Andaluz del Patrimonio Histórico con el concurso de las Comisiones Provinciales del Patrimonio Histórico de la Comunidad y se han desarrollado desde Febrero de 1994 a Marzo de 1995. Entre la documentación generada, es de destacar el punto 3.a. del informe de fecha 13 de Diciembre de 1994 presentado por Da María Morente del Monte representante de la Delegación de Málaga en los referidos Debates Internos.

5. El modelo mejor conocido y más experimentado es el Servicio del Patrimonio Arquitectónico Local de la Diputación de Barcelona, que en la actualidad está necesitado de un cambio o evolución por el agotamiento del propio modelo. No obstante su estudio es muy interesante a la hora de sacar conclusiones.

6. Hasta hace poco tiempo las actuaciones de la Consejería se financiaban de forma directa e imponiéndose el criterio de solo actuar en elementos B.I.C. o incluidos en el C.G.P.H.A. Sin embargo habría que sondear y pulir las demás formas de intervención como convenios, subvenciones y órdenes de ejecución, que clarifican muy bien los distintos niveles de competencia e incluso de responsabilidad, además de otras ventajas de orden económico. A su vez, esto permitiría ampliar el criterio de intervención a la generalidad del Patrimonio Histórico de Andalucía.

7. El art. 27 del Reglamento 4/93 especifica que las actuaciones en los bienes inmuebles sujetos a autorización de esta Administración deben ser informados por las Comisiones Provinciales del
Patrimonio Histórico, concretando el ámbito de bienes del Patrimonio Histórico marcado por el art. 107 de la Ley |/9|. Las actuaciones que de forma directa acomete la Dirección General sobre bienes inmuebles, sujetos a autorización según el art. 19 de la Ley 16/85 y del art. 23 de la Ley |/91, no se someten al informe preceptivo y no vinculante de las referidas Comisiones de forma reglada. (Véase la nota $n^{\circ} 4$ ).

8. Ver nota 7.

9. Hay que tener en cuenta que si se adopta el criterio de poder actuar mediante la fórmula que sea (ver nota 6) no solo se podría actuar en elementos B.I.C. o incluidos en el C.G.P.H.A. y en este caso existen competencias residenciadas en el Delegado Provincial.

10. Así se reconoce en los distintos tipos de Pliegos de Condiciones que de forma provisional vienen funcionando y donde se recoge la figura del coordinador, función que debe ser realizada desde la Delegación Provincial por el Arquitecto jefe del Departamento de Conservación del Patrimonio Histórico. En la línea de este escrito creo llegado el momento de que se trabaje en el sentido de adecuar los referidos Pliegos, tanto a la situación actual, como al desarrollo teórico de las intervenciones llevadas a cabo en estos años.

II. Es en este momento cuando el cuerpo de conocimientos que del elemento se ha obtenido debe pasar a completar y a formar parte del C.G.P.H.A. obteniendo así una mayor utilidad, eficacia y divulgación de la figura creada por la Ley |/9 | para la salvaguarda del Patrimonio Histórico de Andalucía, según se explica en el preámbulo de la referida Ley y cuya lectura periódica es un ejercicio recomendable cuando la práctica diaria nos llega a nublar la visión.

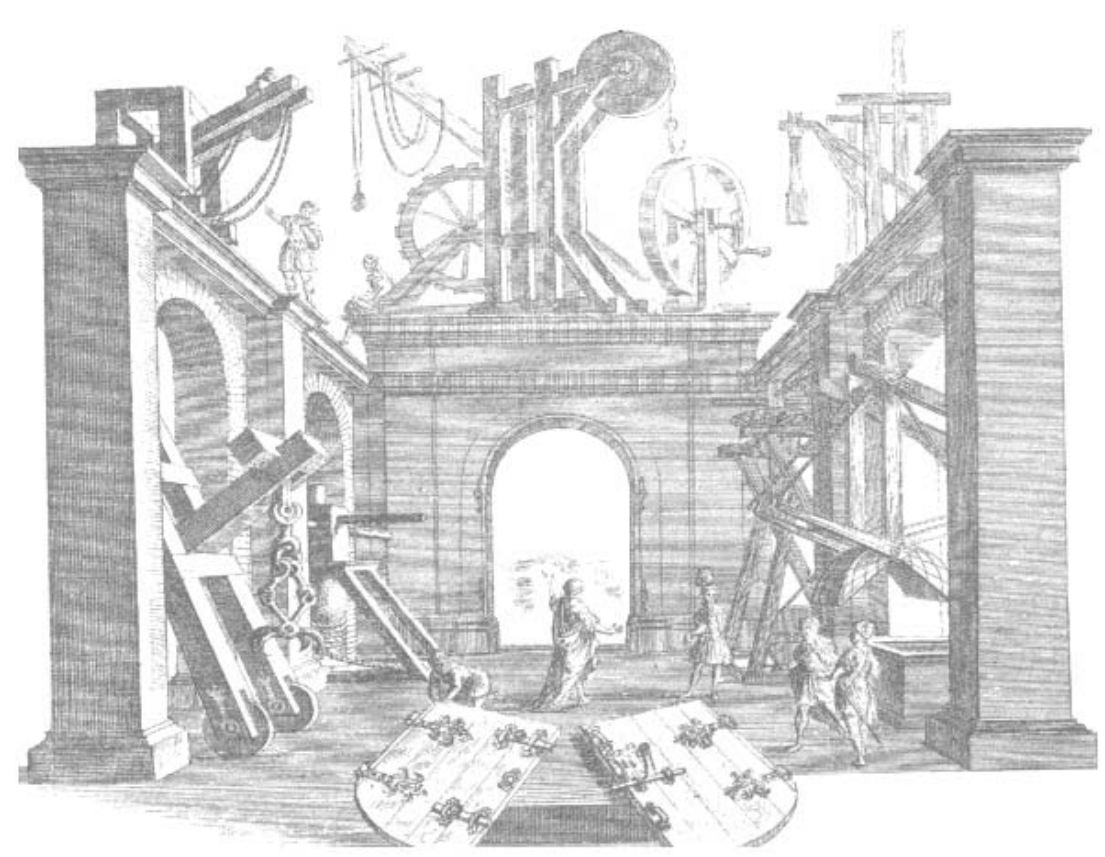

